\title{
Die Grundversorger und ihre «Demo»
}

Nun dürften es bald alle wissen: Die Grundversorger demonstrieren am 1. April 2006 auf dem Bundesplatz in Bern.

Klar, das Datum gibt zum Schmunzeln Anlass ... und zweifellos ist es ein Ziel, einem manchmal recht ernsten, traurigen und wenig phantasievollen Gesundheitssystem und politischen Umfeld auf eine bestimmte Art und Weise zuzuzwinkern!

Dass es sowohl für die Grundversorger wie für die ganze Ärzteschaft um ein hochriskantes Unternehmen geht, ist ebenso klar. Die Botschaft, die man hinüberbringen will, muss durch und durch verständlich, positiv, konstruktiv und hochherzig sein - Aggressionen und Verbitterung dürfen da keinen Platz haben, noch weniger reine Forderungen nach mehr Verdienst. Auf diesem Weg wäre dies ungebührlich für einen Berufsstand, dessen mittlere Einkommen weit über dem Durchschnitt der Gesamtbevölkerung liegen. Sonst droht die Gefahr, dass der Anlass uns komplett falsch ausgelegt wird.

Über den enormen Zorn der Ärztinnen und Ärzte, hier besonders der Grundversorger, hinaus müssen Medien, Politik und Bevölkerung den wahren, tieferen Sinn dieser Demonstration verstehen können:

- Wir Ärztinnen und Ärzte kennen die Medizin, den Berufsalltag, und die Patientinnen und Patienten;

- wir haben Ideen und fundierte Projekte, damit die nötige Weiterentwicklung des Gesundheitssystems stattfinden kann und der Zugang dazu und die optimale Pflege für alle gewährleistet bleiben;

- wir haben konstruktive Lösungsvorschläge für die sich ankündenden Probleme der Finanzierung und des Nachwuchses;

- deshalb müssen die Ärztinnen und Ärzte unbedingt in die Diskussionen über ihre Arbeitsbedingungen und das Gesundheitswesen im allgemeinen mit einbezogen, angehört und respektiert werden;
- die Ärztinnen und Ärzte können und wollen kein Gesundheitswesen billigen, in dem für die am Horizont auftauchenden Probleme nur Alibilösungen gefunden werden, die auf rein ökonomischen Argumenten und politischem Opportunismus basieren.

Offenbar lösen unsere nonkonformistischen Forderungen und Vorschläge, die man nicht einfach als vorgefasste Meinung «klassieren» kann, Überraschung aus: Dabei liegen ihnen doch nur zutiefst menschliche, soziale und politische Überlegungen zugrunde! Dahinter steht auch ein grösserer Zusammenhang. Dieser ist gegeben durch die Perspektive, die sich in den Anforderungen unseres Berufes und in der Würde und den Bedürfnissen der Patientinnen und Patienten bündelt, denen wir tagtäglich in der Vertrautheit der privilegierten Beziehung, die wir mit ihnen haben, begegnen.

Also heisst das ganz konkret: So gut die speziellen Behandlungen oder die Spitzenmedizin für jedermann in der Schweiz versichert bleiben und so gut die Arbeitsbedingungen für Spezialisten sowohl in öffentlichen und privaten Spitälern als auch in freier Praxis garantiert werden müssen, so sehr müssen wir auch die Grundversorgung fördern, denn sie stellt in gewisser Weise den Eckpfeiler in unserem Gesundheitssystem dar.

Wenn wir dies am 1. April zusammen mit den Ärztinnen und Ärzten der Grundversorgung erneut bekräftigen, dann geht es nicht darum, uns an vergangene Privilegien oder an ein überholtes System zu klammern, im Gegenteil: Wir wollen über die Zukunft unseres Gesundheitswesens, über die Grundlagen seiner künftigen Qualität und über unseren Platz in dieser Zukunft sprechen.

Jacques de Haller, Präsident der FMH 